\title{
Atypical Sarcoidosis or Malignant Disease: Case Report of Sarcoid Abdominal Lypmhadenopathy
}

\author{
Vesela Georgieva ${ }^{1}$, Apostol Georgiev ${ }^{1}$, Daniela \\ Chifchiiska $^{2}$
}

1. Department of Gastroenterology, Multiprofile Hospital for Active Treatment "Medline Clinic", Plovdiv

2. Department of Pathology, Multiprofile Hospital for Active Treatment "Medline Clinic", Plovdiv

\begin{abstract}
Sarcoidosis is a chronic multisystem disease, characterized by the presence of noncaseating epithelioid granulomas. The commonest site of affection are the lungs and the the intrathoracic lymph nodes, seen in $90 \%$ of the patients (1). Involvement of intraabdominal viscera is less frequent, it may mimic more common infectious or neoplastic disease. We present a case of a 67year old white woman with isolated abdominal lymphadenomegaly (LAM), with no evidence for gastrointestinal malignancy, who underwent a laparotomy to exclude pancreatic neoplasm or lymphoma. Final diagnosis of abdominal sarcoidosis was obtained after pathological examination.
\end{abstract}

Keywords: sarcoidosis, lymph nodes, gastrointestinal malignancy, biopsy

\section{Background}

Sarcoidosis predominantly affects the lungs and intrathoracic lymph nodes. The first described case of sarcoidosis is in 1869 and belongs to the British dermatologist Johnatan Hutchinson. Sarcoidosis is manifested by the presence of noncaseating granulomas (NCGs) in affected organ tissues. It can affect also liver, spleen, bones, muscles, skin, eyes, the central nervous system, lymph nodes. Isolated extrapulmonary disease occurs in less than $10 \%$ of the cases (1). The incidence is 11 cases per 100000 population in whites, but 34 in 100000 in African Americans. Incidence peaks at the age range from 25 to 35 years. A second peak occurs for women aged between 45 and 65 years (2). 
T-cells play a central role in the development of the disease as they propagate an excessive cellular immune reaction. TNF and TNF-receptors are increased in this disease. A study by Facco et al. suggests that Th17 cells play a role in the pathogenesis and progression of sarcoidosis (3).

\section{Case Description}

We describe a case of a 67-year old white woman. In December 2013 she was referred to a Gastroenterology Department in another hospital because of epigastric pain, nausea, loss of appetite, but no weight loss, no change in the bowel movements, no blood or mucus in the stools. No high body temperature detected. The present state revealed no abnormalities in the respiratory or cardiovascular systems, soft abdominal walls, slightly enlarged liver $-1 \mathrm{~cm}$ under the costal margin. No splenomegaly detected.

Upper and lower gastrointestinal (GI) endoscopies, abdominal ultrasound (US), CT scan and chest X-ray were performed - all were negative for neoplastic diseases. All laboratory tests were within normal limits, except for the GGT - 5 times above th upper limit range, ASAT, ALAT, alkaline phosphatase (AP) - not elevated. A symptomatic treatment was initiated without improvement.

In May 2014 the patient was admitted to our department with progression of the disease, vomiting, consumption of $15 \mathrm{~kg}$ of her weight, elevated macular itchy rash (Fig.1) over the chest and neck, dense liver found 6-7 cm under the costal margin, without splenomegaly.

Fig.1. Macular itchy rash on the chest.

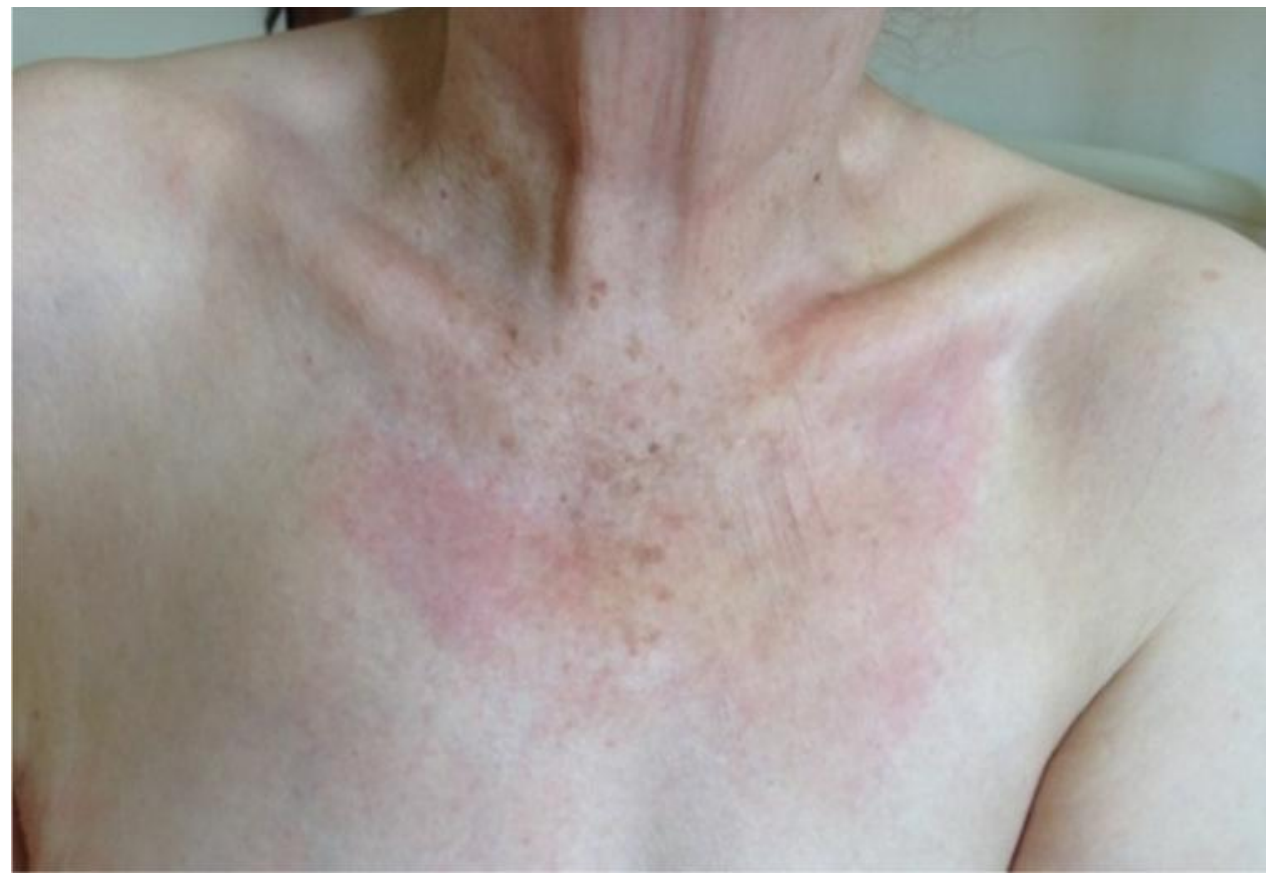


Laboratory tests revealed GGT 160 /0-42/, AP 390 /60-290/, Calcium 3.5/2.1-2.6/, CRP 60 /0.0-6.0/, CA 19-9 705 /0-35/, HbsAg, anti- Hbc total, anti-HCV negative, Quantiferon TB-gold test _- negative for tuberculosis. The following examinations to exclude malignant disease were performed:

- abdominal US- liver cysts, enlarged lymph nodes under the left hepatic lobe

- CEUS - liver cysts, enlarged lymph nodes next to inferior mesenteric vein

- CT scan of the lungs- negative for mediastinal lymph nodes or parenchymal lesions

- CT scan of the abdomen- liver cysts, no pancreatic pathology, no splenomegaly, no enlarged lymph nodes

MRCP - slightly dilated left hepatic duct, no pathological changes in the pancreas, borderline retroperitoneal lymph nodes

Full gynaecological work-up - negative for neoplastic disease.

Because of the clinical presentation, altered liver function, elevated tumor marker $(20 \mathrm{~N})$ and controversial data for enlarged intraabdominal lymph nodes, but without evidence of Gl malignancy, we referred the patient for explorative laparotomy.

Surgical findings:

- enlarged retroduodenal lymph nodes, nodes next to inferior caval vein, hepatic artery and portal vein sized above $2.5 \mathrm{~cm}$

- paraaortic lymph nodes above $3.0 \mathrm{~cm}$.

- hepatomegaly with severe fibrotic changes

- borderline splenomegaly, no parenchymal nodules

- no data for pancreatic malignancy

The pathological examination revealed/result N 1156-59/ 20.05.2014/:

1. Lymph nodes: numerous closely-packed non-necrotizing granulomas, effacing the normal architecture of the lymph nodes. The granulomas contain epithelioid cells and Langhans giant cells. In parts of some of these giant, multinuclear cells there are cytoplasmic calcium inclusions, the so-called Schaumann bodies /asteroid bodies/ (Fig. 2,3).

2. Liver biopsy: The normal architecture of the liver is impaired because of the presence of epithelioid granulomas, without central necrosis, with single Langhans giant cells and several lymphocytes in the periphery (Fig.4). 
Fig. 2. Shaumann body (calcium inclusion in Langhans giant cell) as part of a granuloma.

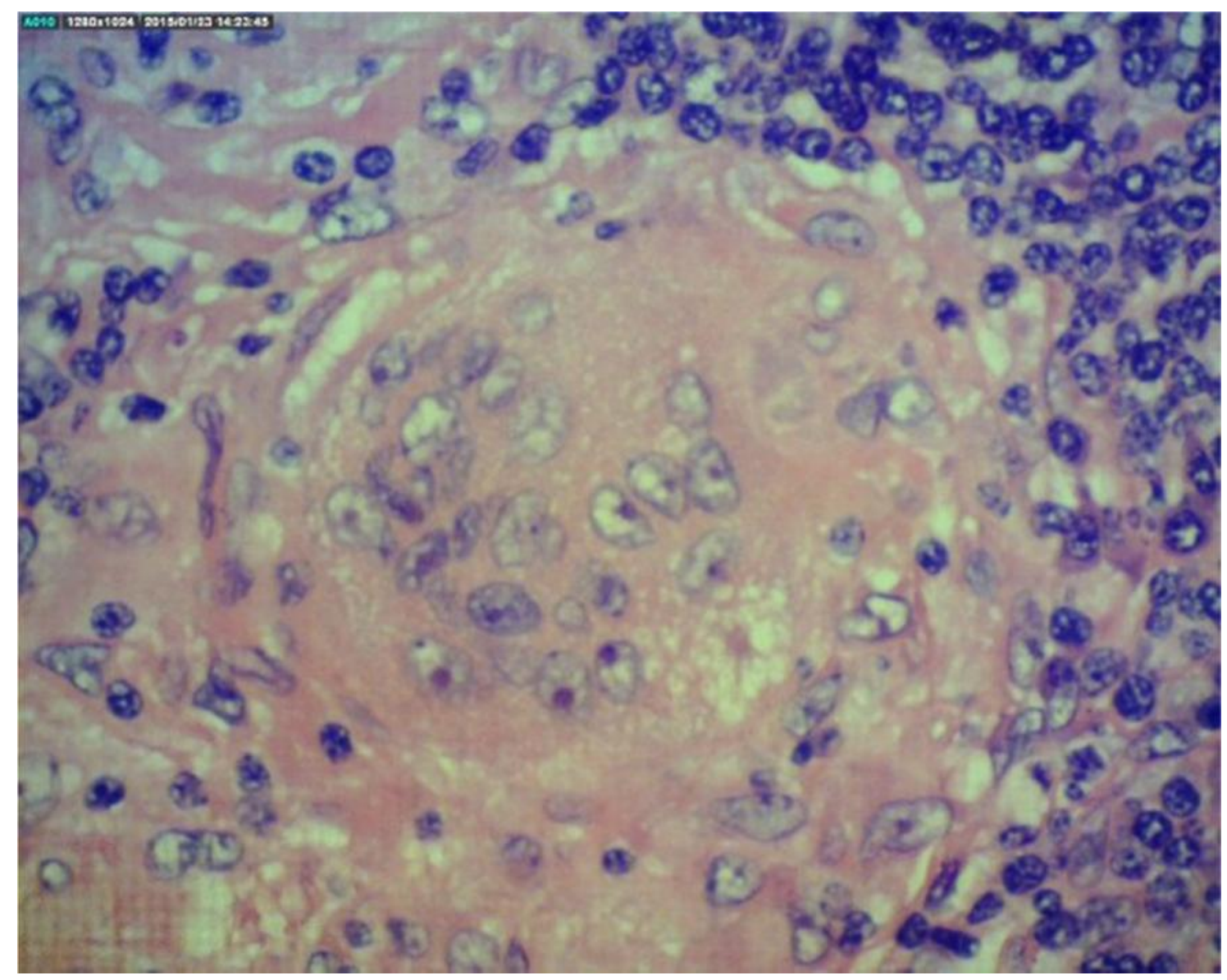


Fig.3. Lymph node with epithelioid granuloma and giant cell.

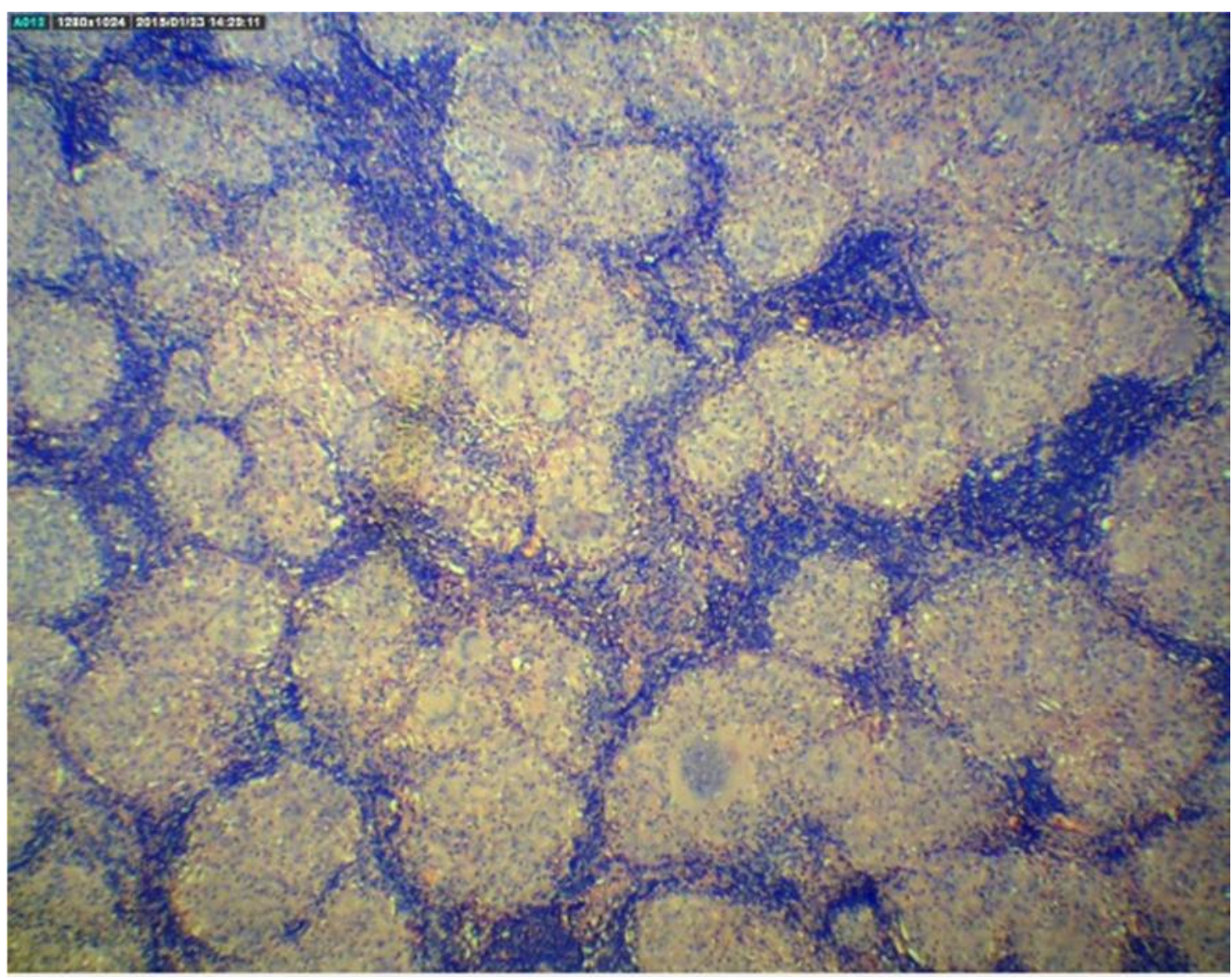


Fig.4. Hepatic granuloma

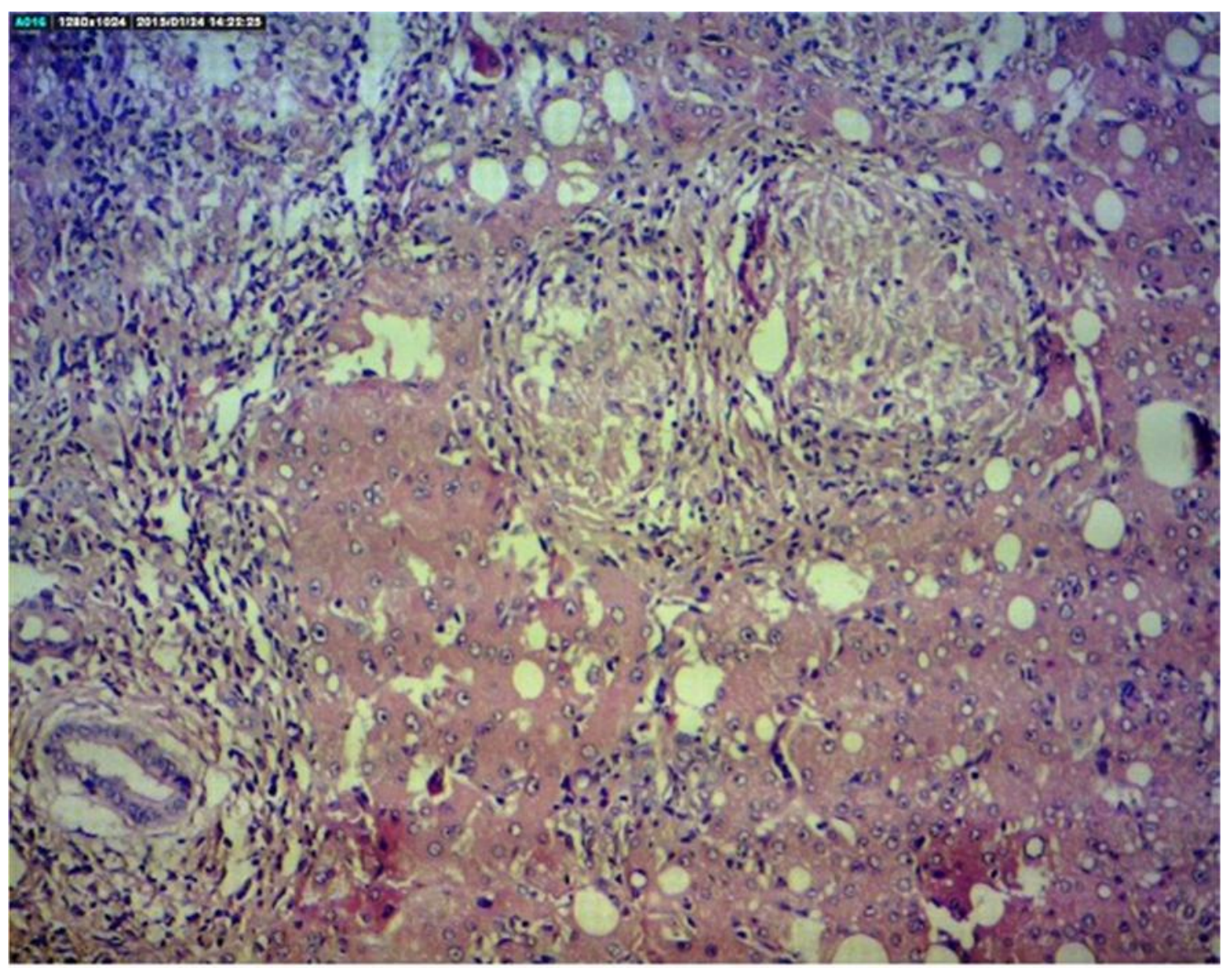

\section{Discussion}

Rare manifestation of sarcoidosis in the gastrointestinal system is hepatic involvement, described in 11.5 $\%$ of 753 patients in ACCESS Study (4). Laboratory evidence of liver dysfunction is seen in 2 to $60 \%$ of patients with alkaline phosphatase being most commonly affected. Symptomatic liver disease is detected in less than $5 \%$. Corticosteroid or immunosuppressive therapies are usually effective in preventing progressive hepatic insufficiency. In rare cases, sarcoidosis may be associated with primary biliary cirrhosis, primary sclerosing hepatitis, Coeliac disease, Crohn's disease, and ulcerative colitis $(4,5)$. Other organs that can be affected are spleen (nodules in $15 \%$, splenomegaly in 25 to60\%), pancreas, gastric and intestine involvement, abdominal lymph nodes.

Abdominal adenopathy (two or more nodes with a short axis above $1 \mathrm{~cm}$ ) is seen in approximately $30 \%$ of patients with sarcoidosis. Extensive adenopathy (above $2 \mathrm{~cm}$ ) is found in about $10 \%$ of the patients (6). In 
comparing the group of patients with sarcoidosis to those with non-Hodgkin's lymphoma, retrocrural nodes were involved significantly more frequently $(70 \%)$ in the patients with lymphoma (6). No significant difference in the frequency of involvement of other nodal stations is found between the two groups (6).

In the above described case with isolated enlarged abdominal lymph nodes, with no data for splenomegaly, no evidence for gastrointestinal malignancy, tuberculosis or other infection, no pulmonary involvement, the question of diagnosing lymphadenomegaly occurred. It was essential to exclude:

1. Pancreas malignancy (abdominal pain, weight loss, elevated cholestatic liver tests, elevated CA 19-9, upper and lower GI endoscopy negative for neoplasia). Because of the absence of pulmonary disease and splenomegaly, sarcoidosis was not considered at the onset of the disease.

2. Immune response to infective agents (important especially when corticosteroid therapy is initiated).

3. Metastases. Differential diagnosis with sarcoidosis is difficult especially when there is no simultaneously involvement of the liver, spleen and lymph nodes (abdominal sarcoidosis "triad").

4. Lymphomas.

\section{Conclusion}

1. In the presence of isolated abdominal LAM and if a diagnosis of systemic sarcoidosis has not been established, usually an intraabdominal lymph node biopsy is needed to exclude malignancy.

2. Needle-biopsy should be considered when a LAM has not been able to be categorized clinically or diagnostically. In about $20 \%$ of cases, the needle- biopsied material is not adequate for citohystological interpretation (7).

3. Surgical biopsy has to be performed when other procedures have failed to elucidate the nature of the abdominal lymphadenomegaly.

\section{References}

1. Giovinale M, Fonnescu C, Soriano A, et al. Atypical sarcoidosis: case reports and review of the literature. Eur Rev Med Pharmacol Sci 2009;13: 1: 37-44.

2. Kamangar N. Sarcoidosis. Medscape 2014.

3. Facco M, Cabrelle A, Teramo A, et al. Sarcoidosis is a Th1/Th17 multisystem disorder. Thorax 2011; 66: 2: 144-150.

4. Moller. D.R. Rare manifestation of Sarcoidosis. Eur respire mon 2005: 32: 233-250.

5. Papadopoulos K, Sjoberg K. Evidence of gastrointestinal immune reactivity in patients with sarcoidosis. J. Intern med 1999; 245: 525-531. 
6. Warshauer DM, Dumbleton SA, Molina PL, et al. Abdominal CT findings in sarcoidosis: radiologic and clinical correlation. Radiology 1994; 192: 93-98.

7. Ghiraddelli M, Jemos V, Gobbi P. Diagnostic approach to lymph node enlargement. Haematologica1999; 84: 242-247.

\section{Corresponding Author}

Vesela Georgieva

Multiprofile Hospital for Active Treatment "Medline Clinic", Plovdiv, Bulgaria

Department of Gastroenterology

37 Phillip Makedonski str., 4000 Plovdiv, Bulgaria

e-mail: medicine@abv.bg 